\title{
Obstructive Sleep Apnea: An overview
}

\author{
Dr. Mala Ram Manohar' ${ }^{1}$ Dr. Juhi Talesra²
}

Professor ${ }^{1}$, PG Student ${ }^{2}$,

Department of Orthodontics, CODS, Davangere.

\section{Abstract:}

Obstructive sleep apnea, is a common disorder that is characterized by repetitive partial or complete cessation of air flow, associated with oxy-hemoglobin desaturation and increased effort to breath.Treatment of obstructive sleep apnea can be divided into four general categories. These include: lifestyle modification, upper airway surgery, oral appliances, and continuous positive airway pressure (CPAP). Although the CPAP provides the most reliable therapeutic modality, it is the most cumbersomeone. Many patients, particularly young non-apneic snorers, find it unappealing, difficult to tolerate, and unacceptable.The only other non-invasive alternative, which can producefavourable results within a short time, is oral appliances.

\section{Keywords: Apnea, Appliances, Oral, Sleep}

\section{Introduction}

Obstructive sleep apnea syndrome (OSAS) is the most common organic sleep disorder, increasingly recognized as a serious public health issue. Sleep apnea, and particularly obstructive sleep apnea, is a common disorder that is characterized by repetitive partial or complete cessation of air flow, associated with oxyhemoglobin desaturation and increased effort to breath.

Middle-aged obese men are at particular risk but the disorder is also present in women and young children. ${ }^{1}$ Because individuals with narrow airways and/or craniofacial anomalies may have increased risk for obstructive sleep apnea/hypopnea syndrome, dentistry can play a pivotal role in the identification and possible treatment of patients with this syndrome. ${ }^{2}$

\section{Etiopathogenesis}

Patients with OSAS have a blockage of the upper airway that may be caused by a variety of anatomic or pathologic conditions. When many of these patients relax and fall asleep, the tongue drops back and actually contacts the posterior and lateral walls of the pharynx. In others, the tongue may not actually contact the walls of the pharynx, but when the patient attempts to inspire, the negative pressure created will suck the tongue and pharynx walls together. In either situation, the patient has an upper airway blockage that prevents air from reaching the lungs. Patients with OSA invariably exhibit severe, chronic, loud snoring. ${ }^{3}$

\section{Classification}

Sleep apnea is classified as central, obstructive, or mixed, and it may be mild, moderate, or severe.

a. Central sleep apnea: It is characterized by the patient's having a loss of oxygen to the lungs caused by the respiratory (chest) muscles making no attempt to breathe as a result of a central nervous system disorder.

b. Obstructive sleep apnea: The respiratory muscles attempt to inspire, but a blockage in the upper airway prevents air from reaching the lungs (Fig. 1).

c. Mixed: A patient with a combination of these problems is said to have mixed apnea. Oral appliance therapy cannot address central and mixed sleep apneas; therefore, this article addresses only OSA, its diagnosis, and its treatment.

\author{
Corresponding Author: \\ Dr. Mala Ram Manohar \\ Professor, \\ Department of Orthodontics, \\ College of Dental Sciences, \\ Davangere-577004. \\ Email:rammanoharmala@gmail.com \\ Mobile: 9741111891
}

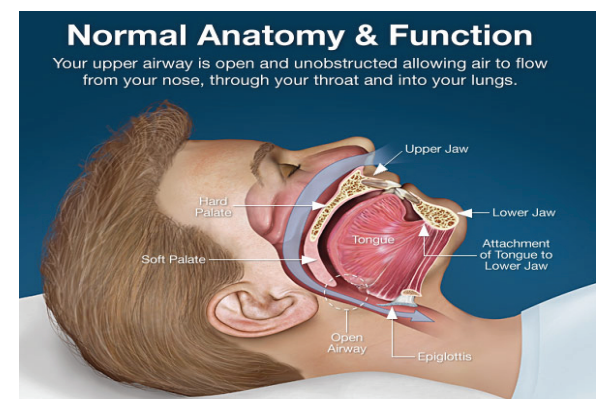

Fig. 1 Obstructive sleep apnea. (Courtesy: http://trynuvigil.com ) 


\section{Clinical features}

Among other consequences of sleep apnea, excessive daytime sleepiness (EDS), cognitive impairment, impaired ability to operate a motor vehicle and an increased automobile accident rate have been recently documented. ${ }^{4}$

Headaches occurring during the night or early morning. Patients with headache also report more daytime symptoms like fatigue, tiredness, or sleepiness. These symptoms contribute to their reduced quality of life.Sleep apnea patients have constricted upper airways that increase the pharyngeal resistance during inspiration. This, in turn, necessitates an increase in pharyngeal dilator muscle contraction to maintain airway potency.

\section{Diagnosis}

OSA may be mild, moderate or severe, depending on the AHI (apnea hypopnea index). An index of fewer than 5 episodes per sleep hour is considered normal, 10 to 20 episodes per hour is mild, 20 to 40 is moderate and more than 40 is severe. $^{5}$

\section{Methods based on clinical data}

History: snoring or witnessed apneas, age, gender Examination: hypertension, body mass index, neck circumference, pharyngeal volume

Epworth sleepiness scale6 is a validatedquestionnaire which indicates a patient's level of day time sleepiness. Scores range from 0 to 3 , and measurethe likelihood of the patient dozing off while watchingtelevision, driving, or reading:

$$
\begin{aligned}
& 0=\text { would never doze }, \\
& 1=\text { slight chance of dozing, } \\
& 2=\text { moderate chance of dozing,and } \\
& 3=\text { high chance of dozing. }
\end{aligned}
$$

\section{Methods based on physiologic testing (Attended or unattended, at home or in laboratory)}

These include Polysomnography, partial-time polysomnography, split-night studies, day nap studies, partial-channel polysomnography, cardiorespiratory sleep study, respiratory sleep study, oximetry.

\section{Polysomnography (PSG)}

A PSG is a physiologic study, usually attended by a trained technologist performed for at least 6 hours during a patient's normal sleep hours. The study records sleep staging and other physiologic variables. Sleep staging includes electroencephalography (EEG), electro-oculography (EOG), and electromyography (EMG). Other physiologic parameters and variables that may be measured include ECG monitoring, airflow, respiratory effort, gas exchange (by oximetry, transcutaneous monitors, or end-tidal gas analysis), limb muscle activity, extended EEG, penile tumescence,gastroesophageal reflux, continual blood pressure monitoring, snoring, and body position.

Video monitoring is recorded for each patient to distinguish better among potential abnormal sleep behaviours including nightmares, nocturnal seizures, or rapid-eye-movement (REM) sleep behavioural disorder. The PSG is scored in separate 30 -second epochs by a registered technologist, then interpreted by a board-certified poly somnographer. Each 30-second epoch is assigned a sleep stage based on published normal patterns.

Sleep Heart Health Study, in 1995 defined an apnea as "decrease of airflow amplitude below $25 \%$ of 'baseline' amplitude lasting for at least 10 seconds. Hypopnea is defined a decrease of airflow amplitude below a $70 \%$ of 'baseline' amplitude, lasting for at least 10 seconds.

The PSG summary report usually describes the overall RDI (Respiratory Disturbance Index), the RDI while supine and the RDI while in REM sleep, and the lowest oxygen desaturation. Sleep architecture is displayed as a graph through the night, termed a hypnogram.

\section{Treatment}

The proposed management of the patient's sleep disorder is measures that may be undertaken include weight loss, nutritional counselling, exercise, the use of a special cervical pillow, smoking cessation, alcohol counselling, or the alteration of the patient's sleep position.

In most instances the dentist involved in the management of the sleep-disordered patient focuses on the management of a breathing-related disorder. The use of an appliance to advance the mandible or some form of a surgical procedure comprises the most significant component of the treatment. ${ }^{2}$

Steps in treatment plan are behaviour modifications, medications, continuous positive airway pressure therapy (Fig. 2), oral appliances (Fig. 3\&4), and surgical treatment. 


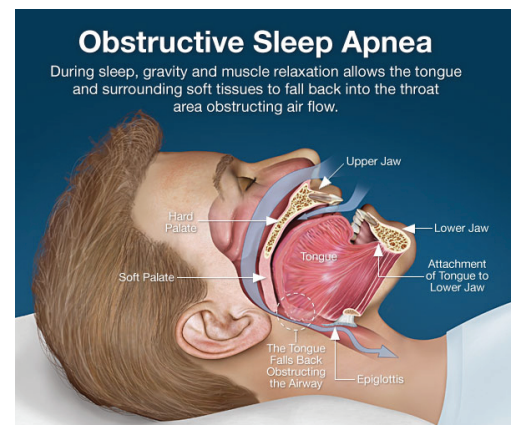

Fig. 2 Continous positive pressure therapy (Courtesy: http://trynuvigil.com ${ }^{15}$ )

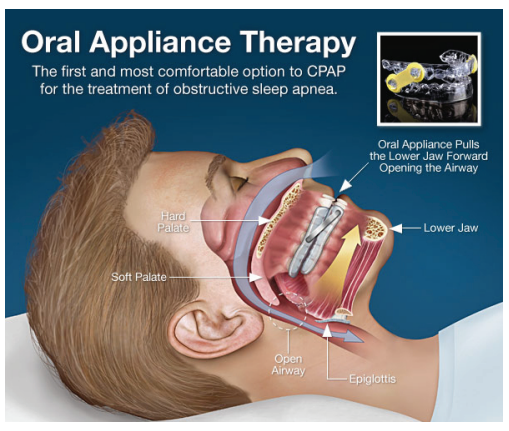

Fig. 3 Oral appliance therapy (Courtesy: http://trynuvigil.com ${ }^{15}$ )
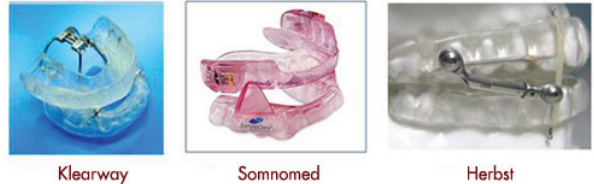

Herbst

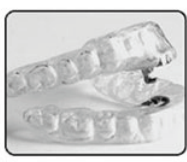

Tap

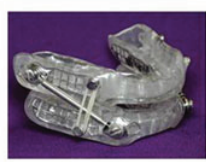

Suad

Fig. 4 Types of oral appliances for obstructive sleep apnea (Courtesy: http://trynuvigil.com ${ }^{15}$ )

\section{Oral appliances}

Dentists must not become the primary care providers for these patients or attempt to treat a medical problem with an oral appliance without a proper diagnosis, which usually requires a sleep study and can only be diagnosed by a physician. Oral devices are basically thermoplastic materials withretainers and supports and are usually custom made. The most commonly used oral devices are:

a. Mandibular repositioning or advancement devices (MRD/MAD) e.g., Herbst appliance ${ }^{7 /}$ snoreguard $^{8} /$ silencer. $^{9}$

b. Tongue repositioning or retaining devices (TRD), e.g. SnorEx. ${ }^{10}$

c. Soft-palate lifters. ${ }^{11}$

d. Tongue trainers. ${ }^{11}$

e. A combination of oral appliance and continuous positive airway pressure CPAP in the newproducts deliver pressurized air directly into the oralcavity. ${ }^{12}$

\section{Indications}

(i) Oral appliances are indicated for use in patients with primary snoring or mild OSA who do not respond or are not appropriate candidates for treatment with behavioural measures such as weight loss or sleep position change;

(ii) Patients with moderate to severe OSA should have an initial trial of nasal CPAP because greater effectiveness has been shown with this intervention than with the use of oral appliances;

(iii) Oral appliances are indicated for patients with moderate to severe OSA who are intolerant of, or refuse treatment with, nasal CPAP. Oral appliances arealso indicated for patients who refuse or who are not candidates for tonsillectomy and adenoidectomy, cranial facial operations or tracheostomy. ${ }^{13}$

\section{Mode of action}

The basic mode of function of these oral appliances is to prevent the tongue from approaching the posterior wall of the pharynx and causing an obstruction (Fig 2). This posterior movement of the base of the tongue is minimized or prevented by use of either a tongue retaining device (TRD) or a mandibular advancement device (MAD), both of which are comprised of concomitant maxillary and mandibular appliance components.Oralappliances improve the blood oxygen saturation levels as they relieve apnea in 20-75\% ofpatients. They reduce the apnea-hypopnea index (AHI) by $50 \%$ or to $<10$ events per hour. Oralappliances also reduce the AHI to normal in $50-60 \%$ patients. $^{14}$

\section{Limitations of oral appliances}

Mandibular protrusion devices should only be used when a patient has at least 8 teeth in each arch and is able to demonstrate a mandibular protrusion of at least $5 \mathrm{~mm}$ and a bite opening of greater than $25 \mathrm{~mm}$. Appliances may be used in combination with partial dentures that replace no more than 4 teeth. Totally edentulous patients are usually not good candidates for mandibular repositioners, but tongue retaining devices may be used in edentulous patients, although these appliances only have FDA clearance for snoring, not OSA. Whereas slight TMJ discomfort can be relieved with forward mandibular positioning, OSA patients who present with more severe TMJ pain probably are not good candidates for treatment with mandibular protrusion appliances.Patients with significant bruxism can frequently damage mandibular protrusion devices and thus make this treatment approach costly and inefficient, while very obese patients, with some exceptions, are best treated by other means than mandibular protrusion. 


\section{Conclusion}

Obstructive sleep apnea is one of the important cause for reduced or impaired quality of life. Along with proper physician consultation and correct diagnosis of the cause and severity of the sleep disorder dentist can help these patients give a better life, as Oral appliances are one of the preferred and acceptable treatment option in the management of sleep apnea syndromes.

\section{References}

1. Strobel RJ, Rosen RC. Obesity and weight loss in obstructive sleep apnea: A critical review. Sleep 1996; 19: 104-15.

2. Epstein LJ, Kristo D, Strollo PJ Jr. Clinical guideline for the evalu $\neg$ ation, management and long-term care of obstructive sleep apnea. J Clin Sleep Med 2009; 5: 263-76.

3. Schwab RJ, Gupta KB, Gefter WB, Metzger LJ, Hoffman EA, Pack AI.Upper airway soft tissue anatomy in normal and patients with sleepdisordered breathing: Significance of the lateral pharyngeal walls. AmJ RespirCrit Care Med 1995; 152: 1673-89.

4. Horne JA, Reyner LA. Sleep related vehicle accidents. BMJ 1995; 310: 565-7.

5. Raphaelson MA, Alpher EJ, Bakker KW, Perlstrom JR. Oral appliance therapy for obstructive sleep apnea syndrome: Progressive mandibular advancement during polysomnography. Cranio 1998; 16: 44-50.

6. Johns MW. A new method for measuring daytime sleepiness: The Epworth sleepiness scale. Sleep 1991; 14: 540-5.

7. Clark GT, Arand D, Chung E, Tong D. Effect of anterior mandibular positioning on obstructive sleep apnea. Am Rev Respir Dis 1993; 147: 624-9.
8. Ferguson KA, Ono T, Lowe AA, al-Majed S, Love LL, Fleetham JA. A short term controlled trial of an adjustable oral appliance for the treatment ofmild to moderate obstructive sleep apnoea. Thorax 1997; 52: 362-8.

9. Schmidt-Nowara WW, Meade TE, Hays MB. Treatment of snoring and obstructive sleep apnea with a dental prosthesis. Chest 1991; 99: 1378-85.

10.Schonhofer B, Stoohs RA, Rager H, Wenzel M, Wenzel G, Köhler D. A new tongue advancement technique for sleep -disordered breathing: Side effects and efficacy. Am J Respir Crit Care Med 1997; 155: 732-8.

11.Schlosshan D, Elliott MW. Sleep, part 3: Clinical presentation and diagnosis of the obstructive sleep apnoea hypopnoea syndrome. Thorax 2004; 59: 347-52.

12.Hart NT, Duhamel J, Guilleminault C. Oral positive airway pressure by the OPAP dental appliance reduces mild to severe OSA. Sleep Res 1997; 26: 371.

13.Practice parameters for the treatment of snoring and obstructive sleep apnea with oral appliances. American Sleep Disorders Association. Sleep 1995; 18:511-3.

14.Liu YH, Lowe A, Fleetham J, Park YC. Cephalometric and physiological predictors of the efficacy of an adjustable oral appliance for the treatment of obstructive sleep apnea. Am J Orthod Dentofac Orthop 2001; 120: 639-47.

How to cite this article: Manohar MR, Talesra J. Obstructive Sleep Apnea: An overview. CODS J Dent 2015;7: 19 - 22

Source of support: Nil. Conflict of interest: None Declared. 